\title{
Otimização Dinâmica Evolucionária para Despacho de Energia em uma Microrrede usando Veículos Elétricos
}

\author{
Marcos Antonio Alves* \\ Lucas Vieira Vilas Boas de Almeida*** \\ Tamires Martins Rezende* \\ Petronio Candido de Lima e Silva* \\ Carlos Alberto Severiano Jr. * Rodrigo Silva ** \\ Frederico Gadelha Guimarães ${ }^{* * *}$ \\ * Programa de Pós-Graduação em Engenharia Elétrica - Universidade \\ Federal de Minas Gerais - Av. Antônio Carlos 6627, 31270-901, Belo \\ Horizonte, MG, Brasil (e-mail: marcosalves@ufmg.br, \\ tamiresrezende@ufmg.br, petronio.candido@ifnmg.edu.br, \\ carlos.junior@ifmg.edu.br). \\ ** Departamento de Computação, Universidade Federal de Ouro Preto, \\ Ouro Preto,Brasil, (e-mail: rodrigo.pedrosa@ufop.edu.br) \\ *** Machine Intelligence and Data Science (MINDS) Lab, Universidade \\ Federal de Minas Gerais - Av. Antônio Carlos 6627, 31270-901, Belo \\ Horizonte, MG, Brazil (e-mail: lucas.vvba@gmail.com, \\ fredericoguimaraes@ufmg.br)
}

\begin{abstract}
This paper presents a method for the power dispatching problem in a smart park that uses plug-in electric vehicles as storage units. The objective is to find the best energy storage planning for 10 vehicles over one day ( 24 hours), in order to minimize the total cost of energy bought from the grid by the smart park. An evolutionary algorithm was applied to solve the problem, considering possible alterations of arrival/departure times of vehicles. At each hour the algorithm was reinitialized and updated values for grid electricity prices, energy needs and solar energy were given by means of a fuzzy time-series model. The results presented an average cost of $\$ 21,576$ (20 executions) and average time of 6.45 seconds. The proposed approach was able to join sucessfully with time-series model, dynamic optimization and evolutionary algorithms to solve a problem of power dispatching.

Resumo: Este artigo apresenta uma proposta para o problema de despacho de energia em um estacionamento inteligente que usa veículos elétricos como unidades de armazenamento. $\mathrm{O}$ objetivo principal é encontrar o melhor planejamento de armazenamento de energia para 10 veículos ao longo de um dia (24 horas), a fim de minimizar o custo total da energia comprada da rede pelo estacionamento. Um algoritmo evolucionário foi aplicado para resolver o problema, levando em consideração possíveis alterações nos horários de entrada/saída dos veículos. A cada hora o algoritmo era reinicializado e valores atualizados para os preços da energia, necessidade de energia e energia solar eram gerados por um modelo de séries temporais fuzzy. Os resultados apresentaram um custo médio de $\$ 21.576$ (20 execuções) e tempo médio de 6.45 segundos. A abordagem proposta foi capaz de combinar eficazmente modelos de séries temporais, otimização dinâmica e algoritmos evolucionários para resolver um problema de despacho de energia.
\end{abstract}

Keywords: Energy dispatching; smart park; plug-in electric vehicles; energy pricing; renewable energy generation; batteries; evolutionary algorithm; dynamic optimization; Differential Evolution algorithm.

Palavras-chaves: Despacho de energia; estacionamento inteligente; veículos elétricos; preço de energia; geração de energia renovável; baterias; algoritmo evolutivo; otimização dinâmica; algoritmo de Evolução Diferencial. 


\section{INTRODUÇÃO}

Vários problemas práticos estão sujeitos a mudanças ao longo do tempo. Um problema dependente no tempo, timedependent ou time-varying, somente é diferente de um problema estático se este for resolvido de uma maneira dinâmica. Isto é, o algoritmo para sua resolução precisa levar em consideração as mudanças que ocorrem durante o processo de otimização à medida que o tempo passa (Nguyen et al., 2012).

É sabido que todo problema real de otimização vai expressar algum grau de incerteza. A incerteza implica na imprevisibilidade do resultado da otimização, uma vez que a solução encontrada em cada caso pode ser muito variável. Para isso, o algoritmo para resolução deve ser capaz de lidar com esta dinamicidade e encontrar boas soluções em diferentes etapas do problema (Woldesenbet e Yen, 2009; Nguyen et al., 2012; Rahman et al., 2016).

Para Woldesenbet e Yen (2009) e Nguyen et al. (2012), esta classe de problemas são resolvidos online (Karp, 1992) por um algoritmo evolucionário, como o de Evolução Diferencial (ED, do inglês Differencial Evolution) (Storn e Price, 1997). O ED possui funcionamento muito similar aos Algoritmos Genéticos (AG), porém com os operadores invertidos: primeiro realiza-se a mutação para em seguida executar o cruzamento. Além disso, ele utiliza um vetor de diferenças para perturbar o vetor população.

Tikader e Ganguly (2014) utilizaram o ED para resolver um problema dinâmico de alocação de energia. Diferentes números de veículos elétricos foram simulados no problema (30, 300 e 1000). A função objetivo consistia na maximização da carga dos veículos na rede. O tempo de carregamento, preço da energia e estado da carga foram considerados, bem como a saída do veículo antes do horário previsto. Apesar dos resultados positivos alcançados, os autores não consideraram outras características dinâmicas ou sustentáveis como geração de energia solar, variação nos preços da energia de hora em hora e utilização de memória para manter a diversidade ao longo das gerações.

Muitas abordagens são capazes de fornecer boas previsões de dados ao longo do tempo como preços e demanda de energia elétrica, bem como para geração energia sustentável. Entre estas abordagens estão os modelos baseados em séries temporais fuzzy. Séries temporais fuzzy são métodos caixa-branca, orientados à dados, não-paramétricos, de alta acurácia e escalabilidade (Silva et al., 2019).

Recentemente, Coelho et al. (2016) abordaram o problema de despacho de energia em uma microrrede em uma versão multiobjetivo. Três objetivos e três critérios foram analisados. Um modelo de Programação Linear Inteira Mista foi utilizado para determinar a solução. Apesar de o modelo ser capaz de encontrar deterministicamente a solução ótima do problema, à medida que a complexidade do problema cresce, i.e. o número de veículos aumenta, o modelo vai perdendo a capacidade de oferecer boas soluções. Nestes casos, os algoritmos evolucionários como o

\footnotetext{
* O presente trabalho foi realizado com o apoio financeiro da CAPES - Brasil e CNPQ
}

ED tem sido de grande auxílio (Plagianakos et al., 2008). Além disso, os autores consideraram apenas uma versão estática do problema e o modelo incluía três veículos, o que torna a aplicação prática da abordagem pouco realista.

Neste contexto, este trabalho buscou implementar uma versão dinâmica para um dos objetivos propostos por Coelho et al. (2016). O objetivo se refere ao balanço de energia em uma microrrede que visa minimizar o custo de energia adquirida da concessionária de distribuição em um estacionamento inteligente ( $\mathrm{SP}$, do inglês smart park) de hora em hora, durante 24 horas, levando em consideração o preço da energia, a energia solar gerada e as necessidades energéticas operacionais.

\section{METODOLOGIA}

\subsection{Definição do Problema}

O problema abordado neste trabalho envolve o balanço de energia de um SP. Ele possui, a priori, uma lista de 10 veículos elétricos (PEV - Plug-in Electric Vehicle). Cada um desses veículos possui um horário de chegada, um horário de saída, o nível de carregamento de sua bateria (SOC, do inglês state of charge) no momento da chegada e o SOC desejado no horário da saída.

O SP precisa de energia para manter suas operações e para carregar as baterias dos PEV estacionados. Ele conta com uma fonte de energia renovável, proveniente de painéis solares. Como premissa, espera-se que a energia renovável gerada não seja suficiente para cobrir todas as demandas necessárias. Assim, vai ser necessário ao SP adquirir energia da concessionária para complementar o que não pôde ser gerado. Este valor da energia comprada flutua ao longo do dia.

O estacionamento também pode somar à sua energia gerada frações das cargas dos $\mathrm{PEV}$ estacionados. Isto quer dizer que em um determinado momento em que o preço da energia da concessionária estiver muito elevado e houver PEV que estejam com SOC suficientes para serem recarregados posteriormente, ao invés de adquirir a energia com preço muito elevado, o SP pode retirar um determinado percentual da carga dos PEV e reabastecê-los até o valor esperado quando a energia estiver mais barata.

Desta forma, deseja-se encontrar quanto de energia cada bateria deve receber do, ou fornecer ao, estacionamento, para cada hora, dentro das 24 horas, de forma a minimizar o valor da energia total adquirida da concessionária, levando em consideração a energia renovável gerada, as necessidades energéticas de operação e a quantidade de veículos previstos para estacionar.

O problema de otimização utilizado neste trabalho é uma adaptação da pesquisa de Coelho et al. (2016) (refere-se ao objetivo 1). O modelo implementado neste trabalho é apresentado a seguir na Equação (1) 


$$
\begin{array}{ll}
\operatorname{Min} & \sum_{i=0}^{N} E C_{i} * E B_{i} \\
\text { sujeito à } \quad & E B_{i}=\max \left[E N_{i}-\left(R E_{i}^{s}-\mathbf{x}_{i j}\right), 0\right], \\
& S O C_{j}^{a}+\sum_{i=P E V_{j}^{a}}^{P E V_{j}^{d}} \mathbf{x}_{i j} \geq S O C_{j}^{d}, \\
& 0 \leq S O C_{j}^{a}+\sum_{i=P E V_{j}^{a}}^{P E V_{j}^{d}} \mathbf{x}_{i j} \leq 100
\end{array}
$$

onde

- $\mathbf{x}_{i j}$ indica a carga na i-ésima hora do j-ésimo PEV, $N$ representa o tamanho da janela de tempo, $M$ o número de PEVs, $E C_{i}$ o custo da energia comprada na i-ésima hora, $E B_{i}$ o total de energia comprada na iésima hora, $E N_{i}$ a necessidade de energia operacional na i-ésima hora, $R E_{i}^{s}$ a energia solar gerada na i-ésima hora, $S O C_{j}^{a}$ e $S O C_{j}^{d}$ o SOC do PEV na chegada e saída, respectivamente, e $P E V_{j}^{a}$ e $P E V_{j}^{d}$ o horário de chegada e saída do PEV, respectivamente;

- Considerou-se um intervalo de tempo $(N)$ igual a 24 horas $(i=1, \ldots, 24)$;

- Foi mantido um número fixo de $10 \operatorname{PEVS}(M)$ veículos no estacionamento $(j=1, \ldots, 10)$. Não foram considerados novos veículos no planejamento do estacionamento;

- A função objetivo deseja minimizar o custo total da energia comprada da concessionária. Ela é, basicamente, a quantidade de energia comprada vezes o custo da energia no momento da compra;

- A primeira restrição define o valor da energia comprada de hora em hora. Caso a necessidade energética não for suprida pela total da energia gerada é necessário comprar energia da concessionária;

- A segunda restrição define que o SOC final de cada PEV, após a permanência no SP, deve ser igual ou maior ao SOC inicial e os balanços que ele sofreu enquanto estava estacionado;

- A terceira restrição limita o SOC final, correspondendo aos limites das baterias (entre 0 e 100 por cento);

- Os limites que cada carga poderia sofrer era de 35 pontos percentuais positivos (veículo está sendo carregado) ou negativos (está sendo utilizada energia do veículo), como em Coelho et al. (2016). Ou seja, se o SOC de uma bateria em uma determinada hora for de $50 \%$, a carga máxima que ela poderia atingir na hora seguinte seria de $50 \%+35 \mathrm{pp}=85 \%$ (ou $15 \%$, no caso de uma descarga), por exemplo. Caso um PEV desejasse um SOC para a saída além do que era possível carregar, o valor era ajustado para esse carregamento máximo.

Em síntese, a modelagem diz respeito a aplicação de um algoritmo dinâmico evolucionário ao problema do balanço de energia de um SP, considerando 10 PEV e um intervalo de 24 horas seguidas. Como a entrada e saída dos veículos e suas respectivas necessidades por carga e tempo de permanência no estacionamento, a reinicialização do algoritmo se deu em relação às horas. Logo, computou-se
24 reinicializações do algoritmo (1 a cada hora) e levando para as futuras gerações uma população com boa avaliação de desempenho como memória explícita (Branke, 1999) em busca da melhor configuração. A Tabela 1 sumariza as informações de horários e cargas das baterias dos veículos previstos.

Tabela 1. Configuração inicial dos PEV, considerando horário de chegada previsto e real, horário de saída previsto e real e SOC de entrada e SOC de saída

\begin{tabular}{ccccc}
\hline PEV & Chegada $(\mathrm{h})$ & Saída $(\mathrm{h})$ & SOC $^{a}(\%)$ & SOC $^{d}(\%)$ \\
\hline 1 & $20 \mid 20$ & $21 \mid 21$ & 21 & 56 \\
2 & $6 \mid 7$ & $10 \mid 10$ & 14 & 80 \\
3 & $3 \mid 5$ & $20 \mid 18$ & 40 & 67 \\
4 & $14 \mid 14$ & $24 \mid 24$ & 8 & 41 \\
5 & $8 \mid 8$ & $9 \mid 9$ & 47 & 56 \\
6 & $17 \mid 16$ & $24 \mid 24$ & 8 & 41 \\
7 & $15 \mid 13$ & $24 \mid 20$ & 79 & 98 \\
8 & $7 \mid 8$ & $24 \mid 24$ & 62 & 98 \\
9 & $8 \mid 10$ & $24 \mid 24$ & 62 & 98 \\
10 & $1 \mid 1$ & $4 \mid 4$ & 79 & 95 \\
\hline
\end{tabular}

\subsection{Definição do Algoritmo Evolucionário}

Dado que as mudanças que acontecem no problema é em função das horas do dia, não foi necessário implementar nenhuma estratégia para detectá-las, conforme explicitaram Woldesenbet e Yen (2009); Nguyen et al. (2012). O custo computacional esperado também é baixo, visto que o algoritmo era executado uma vez para cada hora.

O algoritmo ED (Storn e Price, 1997) foi utilizado para a resolução do problema. Algumas configurações do algoritmo são dadas a seguir:

- A população foi uma matriz de três dimensões: 100 indivíduos x 24 horas x 10 veículos estacionados.

- A função de custo foi calculada com base na quantidade de energia necessária para carregar todos os 10 PEVs ao longo do dia. A cada hora define o ajuste a ser realizado em cada veículo. A função é penalizada caso a carga atual somado ao ajuste da hora corrente for menor que 0 ou maior que 100 ( 5 vezes a diferença absoluta ao quadrado). Penaliza-se, também, caso haja um carregamento da bateria superior ao desejado (10 vezes a diferença do excedido ao quadrado). Depois, o balanço total da hora é calculado (volume de energia que o SP tinha disponível menos a energia gasta para funcionamento operacional e aquele utilizado para carregar as baterias). O SOC final é calculado e comparado ao carregamento final desejado. Se não atender, penaliza-se em 100 vezes o valor não entregue (para refletir a necessidade de entregar aos clientes o valor esperado). Se atender, os melhores indivíduos podem ir para as próximas gerações e novas iterações são realizadas.

- A mutação no DE é feita por meio de um vetor de diferenças para perturbar o vetor população. Os passos são o seguinte: a) escolhe-se um vetor-alvo (i.e. indivíduo alvo); b) randomicamente escolhe-se outros dois vetores; c) randomicamente escolhe-se um vetor alvo de mutação; d) faça a diferença entre os dois vetores escolhidos no passo $b$, pondere, e aplique o 
peso da diferenciação; e) gerar o vetor ruído somandose o vetor de diferenças ponderado ao indivíduo alvo de mutação; f) soma-se o vetor ruído ao indivíduo escolhido no passo $a$.

- Cruzamento: para cada dimensão do vetor um valor aleatório é sorteado. Se o valor for menor que a taxa de cruzamento o gene do novo indivíduo virá do vetor de diferenças, caso contrário virá do elemento da população que estiver sendo mutado. Foi utilizada probabilidade de cruzamento um valor aleatório a cada geração entre 0.7 e 0.9 .

- Um dos tratamentos par manter a diversidade neste tipo de problema é a manutenção da diversidade (Nguyen et al., 2012). Por isso, foram utilizadas duas estratégias diferentes para a memória explícita: 1) ela deve conter as 20\% melhores soluções de uma execução do algoritmo, conforme proposto por Branke (1999); e 2) considera também um conjunto das piores soluções, de tamanho $10 \%$ do tamanho total da população, conforme proposto por Louis e Johnson (1997). O restante, 70\%, foi gerado aleatoriamente;

- Como era conhecida a dinamicidade em relação a hora do problema, o algoritmo era reinicializado de hora em hora. Neste caso, como o intervalo de tempo era conhecido previamente não foi necessário tratar este ponto, como sugeriram Nguyen et al. (2012);

- O critério de parada foi o número máximo de gerações. Ele foi fixado em 1000 (número de PEV * 100);

- O processo final de evolução considera os indivíduos com as melhores avaliações (menor custo) para irem para as próximas gerações e considerando os critérios de manutenção de diversidade.

Uma representação do DE aplicado ao problema pode ser visualizado no Algoritmo 1.

\subsection{Previsão das Séries Temporais}

Como explicado anteriormente, a formulação do problema dinâmico levou em consideração três diferentes previsões, conforme ilustrado na Figura 1: preços de energia $(P E)$, necessidades de energia $(E N)$ e energia solar gerada $\left(R E^{s}\right)$.

Para estimar o preço da energia utilizou-se um conjunto de dados históricos obtido no website EirGrid ${ }^{1}$, onde os valores históricos estavam disponíveis para um total de 926 dias (22.224 horas) na cidade de Hong Kong, que possui o preço da energia flutuante ao longo do dia. O instante inicial de execução da instância do problema em análise foi no 100o. dia (240a. hora) do dados disponíveis. A cada execução, obtém-se a predição de 24 passos à frente e é considerada para o algoritmo o valor predito para a hora corrente em análise.

Já para a a geração da energia solar foram utilizados os valores obtidos por Coelho et al. (2016). Era esperado que a energia renovável solar gerada não fosse capaz de atender às demandas de energia do estabelecimento. Se isso ocorrer, claro, não há necessidade do SP comprar energia da concessionária e o valor da função objetivo (que é calculado sobre a energia comprada) será igual a 0.

\footnotetext{
1 http://www.eirgridgroup.com/operations/systemperformanceda$\mathrm{ta} /$ systemdemand/
}

Retorna Custo total de energia comprada para os 10 PEVs

Carrega as predições;

Inicializa a população (zeros);

Enquanto intervalo de tempo $\leq 24$ faça

Verifica se tem dado na memória (20\% best e $10 \%$ worst) e/ou completa população inicial [-35pp $+35 \mathrm{pp}]$

Avaliar cada indivíduo [carga dos 10 veículos ao longo de 24 horas];

Para Repetir até ngen [npev * 100] faça Para cada indivíduo:

Início

Selecionar 3 indivíduos da população

Início Mutação $[$ ind $1+\alpha($ ind $3-i n d 2)]$

Fim

Compara indivíduo com a sua versão

experimental e melhores vão para próxima geração

Calcula o desempenho de cada indivíduo Fim Ajuste dos indivíduos [-35pp +35pp]

Calcula o custo total da energia comprada. Fim

fim

Algoritmo 1: DE dinâmico aplicado ao problema de despacho de energia

Para a necessidade de energia foram gerados valores aleatórios, calculados da seguinte forma: para cada hora do dia, a média dos valores gerados de energia renováveis eram aumentados em $50 \%$ e sofriam uma perturbação dentro do intervalo $[0 \%, 40 \%]$.

Para estimar estes valores, utilizou-se uma seleção de modelos de séries temporais fuzzy utilizando a biblioteca PyFTS (Silva et al., 2018). O modelo que apresentou a menor RMSE (root mean square error) foi o Weighted Multivariate FTS (WMVFTS) proposto em Silva et al. (2019).

A cada reinicialização do algoritmo, os valores para $\mathrm{PE}$, EN e RE eram gerados. O valor gerado para cada uma das séries está representado na Figura 1. A linha pontilhada com marcações de quadrados representa a energia solar gerada. A linha contínua com marcações em círculos abertos representa a necessidade de energia do SP. Por fim, a linha com traços-e-pontos e marcações com asterisco indica a o preço da energia ao longo do dia. Perceba que a concentração maior de energia gerada está entre 10-17 horas, quando a incidência solar está mais elevada. O preço da energia vendida pela concessionária sofre alterações especialmente no final do dia (conhecido como "horário de pico"), por volta das 18 horas. Já a necessidade de energia do SP para manter suas operações possui menos oscilações.

\section{RESULTADOS E DISCUSSÃO}

Os experimentos foram projetados para buscar pela melhor configuração dos PEVs que minimize o custo total de energia comprada da concessionária utilizando uma 


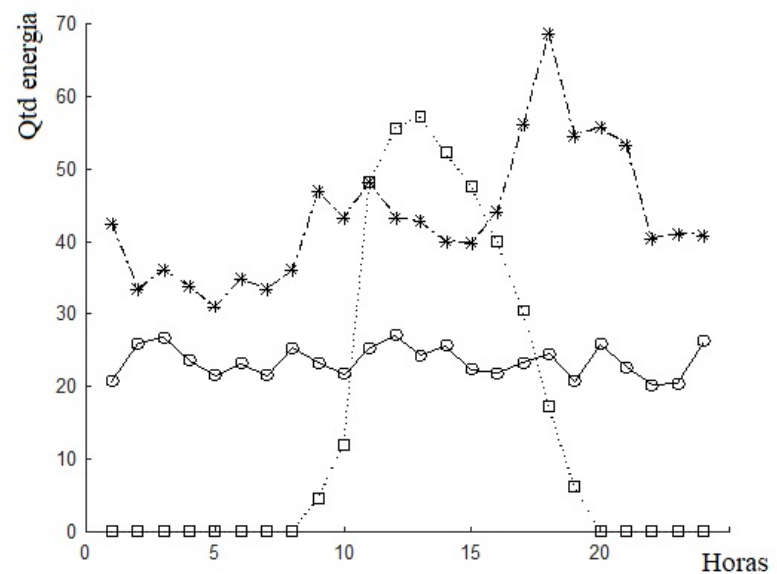

Figura 1. Previsão para o preço da energia vendida pela concessionária, necessidade de energia e energia renovável gerada ao longo das 24 horas

abordagem dinâmica evolucionária para a otimização de uma microrrede.

Os resultados obtidos para as diferentes configurações previamente descritas são exibidos a seguir por meio dos gráficos indicando os SOC's dos veículos ao final do dia. Por motivos de organização, foram escolhidos para serem exibidos na Figura 2 apenas os veículos PEV3, PEV4, PEV6 e PEV7 para representarem as evoluções das soluções e em relação à 1a hora (Fig. 2(a)), 6 a hora (Fig. 2(b)) e 24a hora (Fig. 2(c)). O custo final nestas horas foram, respectivamente, de $\$ 25.370, \$ 24.126$ e $\$ 21.656$. Os valores se alteram ao longo do dia, uma vez que a abordagem proposta consegue encontrar boas combinações ao longo do dia para minimizar o custo total da energia. Esta combinação leva em consideração os horários previstos dos veículos e o preço da energia a ser adquirida pelo SP, incluindo a possibilidade de retirar carga de algum PEV em algum momento para suprir uma outra demanda.

Na Figura 3, por sua vez, são exibidos os dados de todos os veículos (SOC por intervalo de tempo) e o desempenho dos indivíduos ao longo das 1000 gerações. Por meio desta figura é possível perceber, por exemplo, que nas primeiras horas (Fig. 3(a)) o horário de chegada do PEV6 - linha azul contínua - que estava previsto pra chegar as 16 horas (ver Tabela 1) ainda não foi atualizado. A partir das 7 horas este valor foi levado em consideração pelo algoritmo. Esta análise vale para os demais PEVs.

Por fim, foram avaliadas diferentes execuções do algoritmo para checar a consistência do mesmo em relação aos resultados encontrados. Estes testes consideraram o valor final (custo total) da energia comprada e o tempo de execução. Considerando 20 execuções do algoritmo, o algoritmo alcançou, em média, um custo total de $\$ 21.576$ (mínimo $\$ 19.110$, máximo $\$ 23.918$, 1o. quartil $\$ 20.687$, 3o. quartil $\$ 22.405$ e mediana $\$ 21.742$ ). Já o tempo médio de execução foi de de 6.45 segundos (mínimo $\$ 6.01$, máximo $\$ 7.33$, 1o. quartil $\$ 6.16$, 3o. quartil $\$ 6.72$ e mediana $\$ 6.45)$.

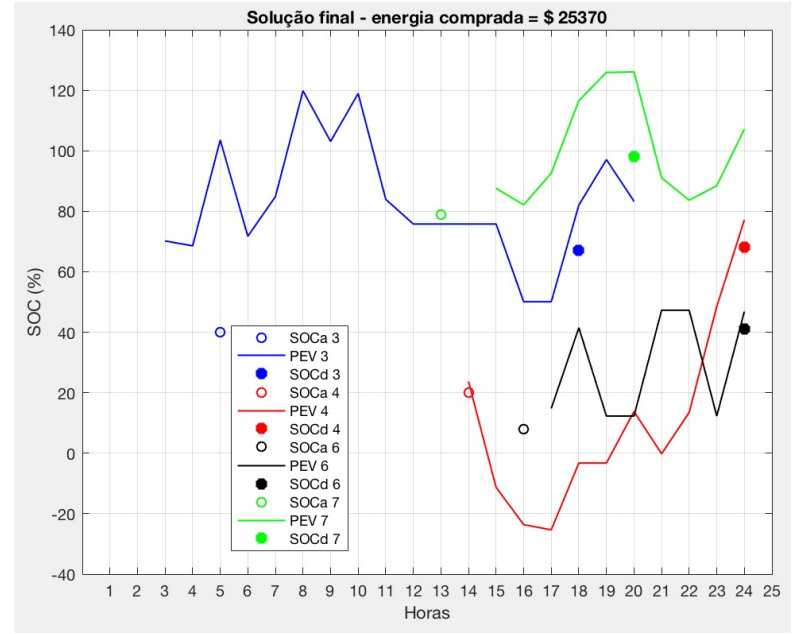

(a) SOC - hora 1

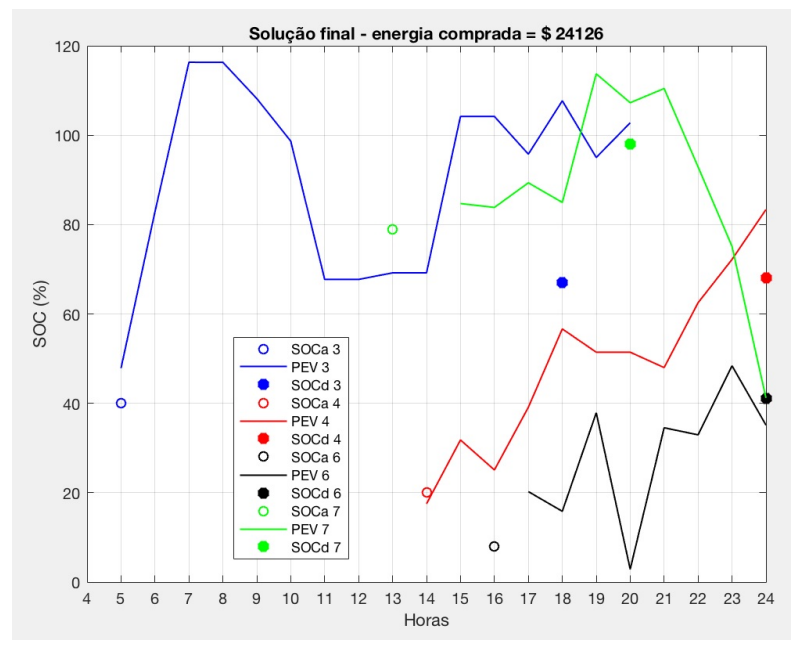

(b) SOC - hora 6

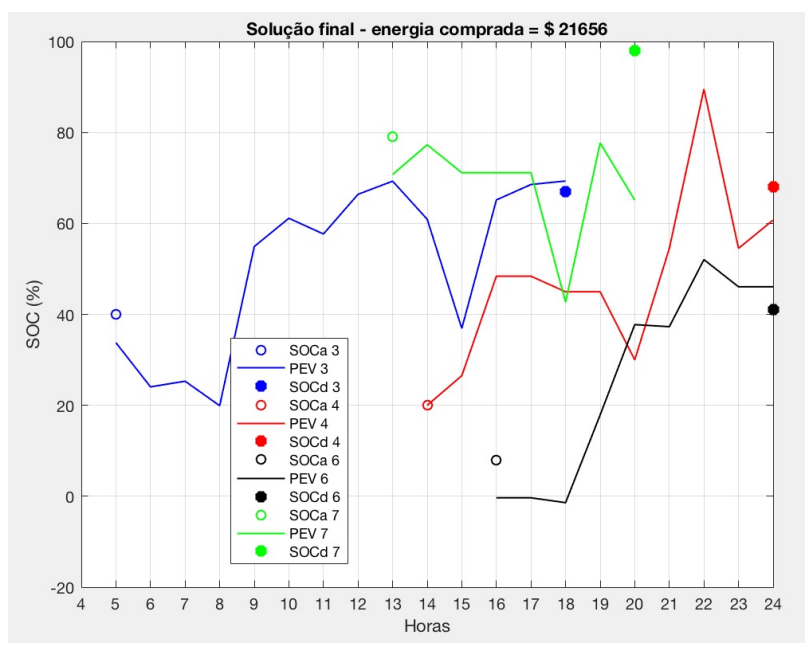

(c) SOC - hora 24

Figura 2. SOC para os PEV 346 e 7 nas horas 1, 6 e 24

\section{CONCLUSÕES}

Este trabalho propôs uma abordagem para o problema de despacho de energia em um estacionamento inteligente. De forma a deixar a formulação do problema mais realista, o preço da energia comprada da concessionaria, a energia 


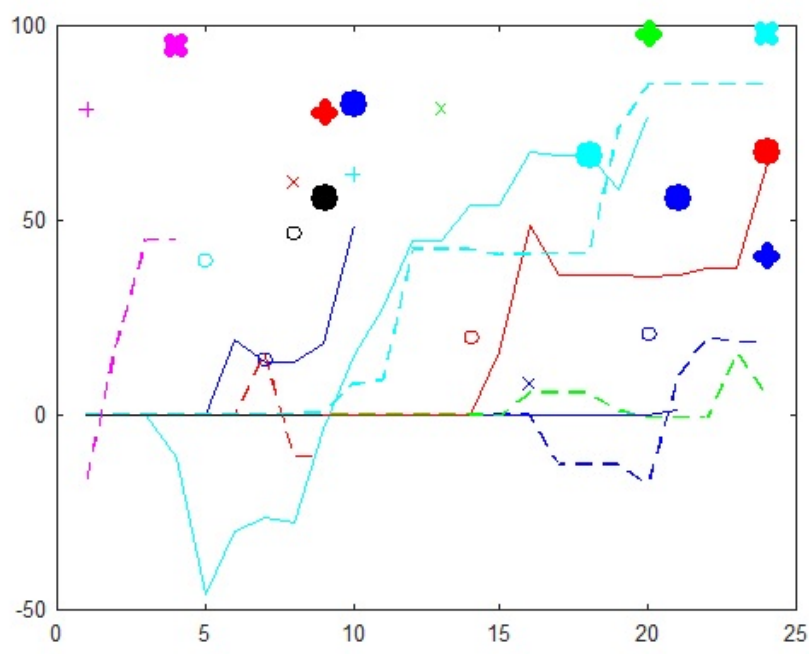

(a) SOC de todos os PEV e informações de chegada e saída

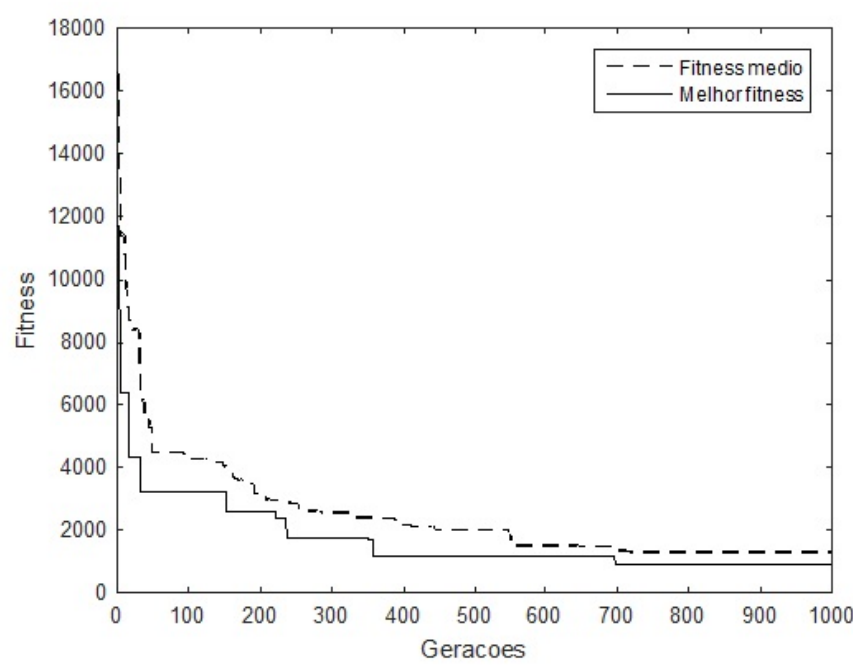

(b) Desempenho dos indivíduos ao longo das gerações

Figura 3. SOC para os 10 PEV com informações hora de chegada/saída e cálculo de desempenho ao longo das gerações

solar produzida, a energia necessária, e o horário de chegada e saída dos PEVs puderam variar dinamicamente.

A metodologia proposta mostrou que é possível combinar modelos de previsão de séries temporais, algoritmos evolutivos e otimização dinâmica para lidar com este tipo de problema. O algoritmo era reinicializado a cada hora e foram encontradas soluções que cumpriram as restrições do problema com um custo médio (em 20 execuções) de $\$ 21.576,00$ executados em uma média de 6.45 segundos.

Apesar de um problema similar ter sido tratado em (Coelho et al., 2016), anteriormente, a dinamicidade de todos os fatores citados acima não foram levadas em consideração. Assim, a continuação deste trabalho pode se dar de diferentes formas, quais sejam: avaliar o desempenho de outros algoritmos evolucionários em face ao problema formulado, incluir previsão eólica e avaliar novos intervalos de tempo (por exemplo, minutos), abordagem multiobjetivo (similar aquela proposta por Coelho et al. (2016)), inclusão de mais veículos no estacionamento e a chegada ou saída inesperada de alguns deles, entre outros.

\section{AGRADECIMENTOS}

O presente trabalho foi realizado com o apoio financeiro da CAPES - Brasil e do CNPq, Conselho Nacional de Desenvolvimento Científico e Tecnológico - Brasil (169392/2017$1)$.

\section{REFERÊNCIAS}

Branke, J. (1999). Memory enhanced evolutionary algorithms for changing optimization problems. In Proceedings of the Congress on Evolutionary ComputationCEC99 (Cat. No. 99TH8406), volume 3, 1875-1882. IEEE.

Coelho, V.N., Coelho, I.M., Coelho, B.N., Cohen, M.W., Reis, A.J., Silva, S.M., Souza, M.J., Fleming, P.J., e Guimarães, F.G. (2016). Multi-objective energy storage power dispatching using plug-in vehicles in a smartmicrogrid. Renewable Energy, 89, 730-742.

Karp, R.M. (1992). On-line algorithms versus off-line algorithms: How much is it worth to know the future? In IFIP congress (1), volume 12, 416-429.

Louis, S.J. e Johnson, J. (1997). Solving similar problems using genetic algorithms and case-based memory. In ICGA, 283-290. Citeseer.

Nguyen, T.T., Yang, S., e Branke, J. (2012). Evolutionary dynamic optimization: A survey of the state of the art. Swarm and Evolutionary Computation, 6, 1-24.

Plagianakos, V., Tasoulis, D., e Vrahatis, M. (2008). A review of major application areas of differential evolution. In Advances in differential evolution, 197-238. Springer.

Rahman, I., Vasant, P.M., Singh, B.S.M., Abdullah-AlWadud, M., e Adnan, N. (2016). Review of recent trends in optimization techniques for plug-in hybrid, and electric vehicle charging infrastructures. Renewable and Sustainable Energy Reviews, 58, 1039-1047.

Silva, P.C.L., Lucas, P.O., e Guimarães, F.G. (2019). A distributed algorithm for scalable fuzzy time series. In Proceedings of 14 th International Conference on Green, Pervasive, and Cloud Computing (GPC 2019). Springer Nature Switzerland, Uberlândia. doi:10.1007/ 978-3-030-19223-5_4.

Silva, P.o.C.a.d.L., Alves, M.A.o., Severiano Jr, C.A., Vieira, G.L., Guimarães, F.G., e Sadaei, H.J. (2018). pyfts: Fuzzy time series for python. doi:10.5281/zenodo. 597359. URL https://pyfts.github.io/pyFTS/.

Storn, R. e Price, K. (1997). Differential evolution-a simple and efficient heuristic for global optimization over continuous spaces. Journal of global optimization, 11(4), 341-359.

Tikader, R. e Ganguly, S. (2014). Energy management at municipal parking deck for charging of plug-in hybrid electric vehicles. In 2014 IEEE Students' Conference on Electrical, Electronics and Computer Science, 1-5. IEEE.

Woldesenbet, Y.G. e Yen, G.G. (2009). Dynamic evolutionary algorithm with variable relocation. IEEE Transactions on Evolutionary Computation, 13(3), 500-513. 